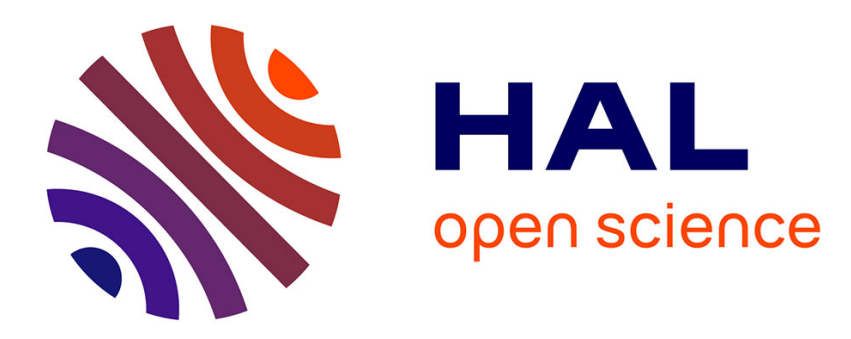

\title{
Les dynamiques organisationnelles liées à la RSE. Le cas de la relation siège-filiale
}

\author{
Julie Bastianutti
}

\section{To cite this version:}

Julie Bastianutti. Les dynamiques organisationnelles liées à la RSE. Le cas de la relation siège-filiale. Revue Française de Gestion, 2014, 40 (240), pp.115 - 132. 10.3166/RFG.240.115-132 . hal-01661758

\section{HAL Id: hal-01661758 https://hal.science/hal-01661758}

Submitted on 26 Jun 2018

HAL is a multi-disciplinary open access archive for the deposit and dissemination of scientific research documents, whether they are published or not. The documents may come from teaching and research institutions in France or abroad, or from public or private research centers.
L'archive ouverte pluridisciplinaire HAL, est destinée au dépôt et à la diffusion de documents scientifiques de niveau recherche, publiés ou non, émanant des établissements d'enseignement et de recherche français ou étrangers, des laboratoires publics ou privés. 


\section{LES DYNAMIQUES ORGANISATIONNELLES LIÉES À LA RSE}

Le cas de la relation siège-filiale

Julie Bastianutti

\section{Lavoisier | Revue française de gestion}

\section{$2014 / 3-N^{\circ} 240$ \\ pages 115 à 132}

\section{ISSN 0338-4551}

Article disponible en ligne à l'adresse:

http://www.cairn.info/revue-francaise-de-gestion-2014-3-page-115.htm

Pour citer cet article :

Bastianutti Julie, « Les dynamiques organisationnelles liées à la RSE » Le cas de la relation siège-filiale, Revue française de gestion, 2014/3 № 240, p. 115-132. DOI : 10.3166/RFG.240.115-132

Distribution électronique Cairn.info pour Lavoisier.

(c) Lavoisier. Tous droits réservés pour tous pays.

La reproduction ou représentation de cet article, notamment par photocopie, n'est autorisée que dans les limites des conditions générales d'utilisation du site ou, le cas échéant, des conditions générales de la licence souscrite par votre établissement. Toute autre reproduction ou représentation, en tout ou partie, sous quelque forme et de quelque manière que ce soit, est interdite sauf accord préalable et écrit de l'éditeur, en dehors des cas prévus par la législation en vigueur en France. II est précisé que son stockage dans une base de données est également interdit. 


\section{Les dynamiques organisationnelles liées à la RSE}

\section{Le cas de la relation siège-filiale}

La recherche ${ }^{1}$ étudie les dynamiques relationnelles entre un siège et ses filiales liées au développement de la RSE. Quelles sont les dynamiques à l'œuvre permettant de comprendre comment se construisent les enjeux de RSE dans un groupe industriel diversifié ? Dans quelle mesure cela fait-il évoluer l'interdépendance des entités? La réflexion théorique porte sur les enjeux stratégiques internes liés au développement de la RSE à partir d'une analyse des modalités de transformation de la relation siège-filiale. Une étude de cas est menée à partir d'un groupe industriel français diversifié, sur la période 20002012. Les résultats montrent une dynamique de centralisation incomplète de la relation siège-filiale et une instabilité organisationnelle qui demeure forte à la fois dans les filiales et dans la relation siège-filiale.

DOI:10.3166/RFG.240.115-132 (C) 2014 Lavoisier

1. L'auteur remercie les rédacteurs invités ainsi que les deux évaluateurs pour leurs remarques constructives. Il remercie également pour leurs précieux commentaires tout au long du processus ayant mené aux premières versions de ce texte : Hervé Dumez, Franck Aggeri, Olivier Borraz, Michel Capron, Cécile Chamaret, Benoît Demil, Christina Garsten, Marie-Rachel Jacob, Xavier Lecocq, Benjamin Lehiany, Felix von Pechmann, les participants au séminaire « Organizing knowledge » (mai 2012, université de Stockholm par le SCORE et le CSO), les participants à la session 27 de la XXII ${ }^{\mathrm{e}}$ conférence de l'AIMS et au $8^{\mathrm{e}}$ congrès du RIODD (juin 2013). L'auteur tient enfin à remercier les acteurs du terrain pour leur confiance et l'accès accordé à cette recherche. Les erreurs sont imputables à l'auteur de ce texte et lui seul. 
$\mathrm{L}$ a responsabilité sociale de l'entreprise (RSE) peut se comprendre comme un domaine d'intervention élargi où les expériences collectives produisent de l'inattendu et révèlent des potentialités nouvelles pour l'action stratégique de l'entreprise (Aggeri, 2011). L'objet de la recherche se concentre sur les dynamiques relationnelles entre un siège et ses filiales liées au développement de la RSE. L'étude des interactions entre une société-mère et ses filiales nous semble une approche pertinente pour saisir les enjeux du développement d'une politique de RSE au sein des groupes industriels diversifiés. En effet, dans ce cas, la diversité des attentes en matière environnementale et sociale reflète la variété des activités industrielles et de services. Cela pose la question de leur intégration et de leur gestion au niveau du siège et induit une modification des équilibres de la relation entre siège et filiale. Quelles sont les dynamiques à l'œuvre permettant de comprendre comment se construisent les enjeux de RSE dans un groupe diversifié, où les pressions sont diversifiées par activité ? Dans la partie théorique, nous revenons sur les enjeux stratégiques internes liés à la RSE dans les groupes industriels avant d'analyser les évolutions possibles de la relation siège-filiale. Cela nous conduit à poser trois questions portant sur l'évaluation de l'interdépendance des entités sous l'effet des enjeux de RSE, le degré de centralisation et décentralisation des relations et l'émergence de nouvelles modalités relationnelles entre les entités. Au niveau empirique, le champ d'investigation est le groupe Bouygues et l'unité d'analyse, l'interaction stratégique entre les différentes entités. Bouygues est un groupe coté en Bourse donc soumis à un impératif légal de reporting extrafinan- cier, et bénéficiant en France d'une notoriété certaine, le rendant sensible aux effets d'image et de réputation. Ses activités sont diversifiées dans la construction (immobilier, bâtiments et travaux publics) et la communication (médias, télécommunications). Ceci permet de mettre en évidence l'impact de la variété même des activités plus que la variable d'extension géographique. Enfin, la présence de plusieurs filiales appartenant à un même secteur industriel permet d'analyser les phénomènes de concurrence ou de coopération entre les filiales. Nous présentons une première série de résultats montrant que la RSE s'accompagne d'une dynamique de centralisation incomplète. Le cas permet de mettre en valeur les problématiques spécifiques aux groupes diversifiés. Les contributions théoriques portent sur la gestion de la relation siège-filiale. La diversité des activités industrielles semble favoriser des modalités de contrôle souple et normatif par un siège qui ne parvient pas à mettre en place une centralisation complète. Quand les enjeux de RSE ont une dimension concurrentielle, ils constituent un facteur majeur d'instabilité dans la relation siègefiliale et filiale-filiale.

\section{I - QUELS IMPACTS DE LA RSE SUR LA RELATION ENTRE LE SIÈGE ET SES FILIALES ?}

Dans un groupe industriel diversifié, la RSE tend à renforcer l'interdépendance entre les entités et remet à l'agenda stratégique la question, au niveau du siège, de la capacité d'orchestration d'un changement stratégique global. Par interdépendance, nous entendons une relation de dépendance réciproque entre les entités. Plus précisément, cela signifie par exemple que le siège 
se trouve dépendant de ses filiales dans la mesure où le groupe, en tant que société cotée, est jugé par les analystes et investisseurs sur les actions environnementales et sociales de ses filiales. La transversalité de certaines problématiques implique la mise en œuvre de procédures concertées et communes qui créent, à l'inverse, des interdépendances de fait entre les entités : certaines filiales n'étant pas initialement sensibles à certaines problématiques le deviennent par effet de «contagion». Cela se traduit par une modification des équilibres de la relation entre siège et filiale et de nouvelles modalités relationnelles.

\section{Les enjeux stratégiques internes de la RSE dans les groupes diversifiés}

Dans les groupes diversifiés, la RSE apparaît comme un défi stratégique et organisationnel en interne. Ce constat constituait déjà le cœur de la réflexion du courant de corporate social responsiveness dans les années 1970, qui questionnait la capacité des firmes à orchestrer le changement stratégique (Ackerman et Bauer, 1976). En effet, la diffusion des structures divisionnelles, fondées sur l'autonomie de chaque entité et un contrôle financier de la part du siège, a eu pour conséquence de faire perdre aux groupes l'expérience de la mise en œuvre de politiques générales qui ne soient pas tournées vers la réalisation d'objectifs financiers. Or, les enjeux de RSE créent de nouvelles interdépendances entre les entités et imposent de réévaluer le besoin de centralisation et décentralisation.

\section{Les enjeux de RSE accroissent l'interdépendance entre entités}

La RSE impose à l'entreprise de prendre en considération une multitude d'enjeux envi- ronnementaux et sociétaux étant de plus en plus rationalisés, c'est-à-dire portés par des acteurs organisés, tant du point de vue des arguments que des actions entreprises pour en justifier l'importance. Ces enjeux sont souvent en concurrence mais doivent faire l'objet d'une égale attention de la part de la firme, au moins pour évaluer la nécessité de les prendre en compte. Le contexte opérationnel s'élargit à la faveur d'un environnement externe, fragmenté et en concurrence, qui génère des conflits de demandes au sein même des organisations (Bromley et Powell, 2012). Ces enjeux sont transversaux, et ce de deux manières. D'une part, un même enjeu peut toucher plusieurs divisions, à des degrés différents. Ainsi, l'empreinte carbone est devenue un sujet pertinent pour toutes les activités, même celles pour qui cela n'apparaissait pas, a priori, comme un point d'attention majeur, par exemple les services reposant sur des prestations intellectuelles ou les médias. En France, la représentation de la diversité (ethnique, sociale, générationnelle) parmi les employés est une question prise au sérieux quel que soit le secteur d'activité. D'autre part, dans un groupe diversifié, un enjeu majeur pour une division peut devenir un enjeu pour chaque filiale, s'il a un fort impact au niveau du groupe et la capacité de susciter l'attention des médias ou des activistes. Afin d'éviter des dommages réputationnels et les effets de spill-over (Yu et Lester, 2008), les enjeux primordiaux pour une division doivent être gérés au niveau du groupe et des autres filiales. Ou, au contraire, des enjeux qui sembleraient anodins au niveau d'une filiale, une fois combinés aux enjeux mineurs des autres activités, peuvent se révéler importants à prendre en compte au niveau du périmètre organisationnel glo- 
bal (Daudigeos, 2009). L'impact sur les différents niveaux de l'entreprise peut être interprété comme l'effet d'un champ de responsabilisation dont les manifestations peuvent également conduire à la formation de nouvelles responsabilités juridiques pour l'entreprise (Bastianutti et Dumez, 2012). Les conséquences de ces nouvelles interdépendances se situent à la fois au niveau organisationnel et stratégique.

\section{Les conséquences de l'interdépendance sur les problématiques organisationnelles}

L'un des apports majeurs d'Ackerman et Bauer (1976) consiste à avoir élaboré un modèle présentant les étapes et défis de la mise en œuvre d'un processus de changement stratégique et organisationnel pour le cas particulier des questions sociales. Ce processus est présenté de façon synthétique et critique par Acquier et al. (2011) qui mettent en avant la prise en compte du temps long de l'action managériale et du rôle de la direction pour donner l'impulsion dans le processus d'identification des enjeux. Ceux-ci sont d'abord traités comme une « question politique », c'est-à-dire avant tout un objet de discours, donnant lieu à des déclarations (en interne et en externe) mais pas encore à des changements de procédures internes, à de nouvelles techniques (de production ou de management). Puis viennent les phases d'intégration dans les processus, et enfin de transformation managériale, pouvant se traduire par un certain nombre de crises et manifestations ouvertes de résistance. Durant la période intermédiaire d'intégration, les experts et les boundary-spanners constituent une catégorie d'acteurs à part, dont l'observation révèle bien des aspects de la vie de l'organisation habituellement cachés par les routines et les règles instituées. En brouillant les règles du jeu et en mettant en évidence les nouveaux défis, ils mettent l'organisation face à ses limites, tant en termes de frontières que de capacités. Il s'agit également de construire un sens à partir de ces changements quand le cadrage n'est pas stabilisé ; les experts fonctionnels doivent remplir ce rôle et leur action a des répercussions à tous les niveaux de l'entreprise.

Cette question de l'expert fonctionnel, pivot entre les différents niveaux de l'organisation, est également une opportunité de discuter le besoin de plus de centralisation ou, au contraire, de décentralisation. L'argument central du modèle de la contingence (Doz et Prahalad, 1984), par exemple, soutient le choix de la décentralisation, les managers opérationnels ayant une meilleure connaissance des problématiques locales. Daudigeos (2009) montre que cela peutêtre contre-productif pour la gestion des enjeux sociaux qui demandent des visions de long terme.

Dans cette optique, dans un groupe industriel où les pressions sont diversifiées par activité, comment évaluer l'interdépendance des entités sous l'effet des enjeux de RSE ?

\section{Quelles modalités d'interaction entre le siège et les filiales ?}

Pour opérationnaliser la question de la centralisation, nous choisissons comme point d'entrée la relation siège-filiale, plus large que celle du rôle de l'expert fonctionnel et permettant d'articuler plusieurs niveaux d'analyse (celui des acteurs, celui de l'organisation contrôlée, celui de l'organisation ayant le contrôle). Quand le siège veut développer de nouvelles activités transverses, touchant l'ensemble des métiers, cela peut 
passer par un regain de centralisation, ou au contraire, on l'a vu, par un mode de gestion décentralisé. Cependant le problème apparaît d'emblée plus complexe. Certaines problématiques de RSE émanent du terrain (activité ou zone géographique particulière) et trouvent un écho au siège. De même, les filiales entretiennent entre elles des échanges. Il s'agit de montrer la variété des modalités d'interactions entre les entités et leurs effets organisationnels et stratégiques.

\section{Les relations siège-filiales : une littérature centrée sur la question du contrôle et de la performance des multinationales}

Les relations entre un siège et ses filiales ont fait l'objet d'une grande attention de la recherche académique qui s'intéresse au management stratégique des multinationales. Ces recherches étudient l'équilibre contrôle-coopération dans le management de la relation entre un siège et ses filiales (Abdellatif, 2011 ; Beddi, 2011 ; Hamilton et al., 1996 ; Martinez et Jarillo, 1991 ; Mayrhofer, 2011 ; Roth et Nigh, 1992). Nohria et Ghoshal (1994) mettent en avant la question de la performance financière du groupe à travers l'efficacité des « corporate control mechanisms ». D'autres se concentrent sur la performance des filiales et ses variations en fonction des modes de contrôle du siège et de l'autonomie des filiales (Doz et Prahalad, 1984 ; Van de Ven et al., 2012).

Certaines recherches s'intéressent moins aux équilibres macro-organisationnels qu'aux équilibres micro, en analysant le management de la relation siège-filiale du point de vue des acteurs (Birkinshaw et al., 2000 ; Nohria et Ghoshal, 1994). Le rôle des individus en position de marginal-sécant (Crozier et Friedberg, 1977) et jouant un rôle d'agent de liaison (boundary-spanners) entre entités met l'accent sur les jeux de frontières, les interfaces et le rôle assigné à ces individus pour ajuster les modalités relationnelles et favoriser la communication (Rao et Sivakumar, 1999 ; Richter et al., 2006 ; Schotter et Beamish, 2011 ; Williams, 2002).

Dans un groupe industriel faisant face à des pressions diversifiées, l'intégration d'une politique RSE est-elle un facteur de centralisation ou de décentralisation?

\section{Les nouvelles modalités d'interactions entre siège et filiales liées à la RSE}

L'étude des relations siège-filiales liées aux enjeux de RSE trouve un écho particulier dans la littérature portant sur les firmes multinationales plus que sur les groupes diversifiés de façon spécifique (AmbaRao, 1993 ; Muller, 2006 ; Pestre, 2011 ; Pinkston et Carroll, 1992 ; Zyglidopoulos, 2002). D'autres recherches s'intéressent aux transferts d'innovation permis par les relations entre un siège et ses filiales étrangères situées dans des pays en développement (Borini et al., 2012; Veugelers et Cassiman, 2004). Enfin, Cruz et al. (2010) mettent en avant le rôle décisif de l'existence d'une structure transverse pour le management de la RSE, comme étant la condition d'intégration de la politique RSE entre le siège et ses filiales. Une telle structure mélange des personnes appartenant aux filiales et au siège, ayant parfois une double fonction (boundary-spanner) permettant de développer des coopérations effectives. Le postulat de l'article est en faveur d'une centralisation se manifestant par une intégration effective. Ceci suppose un certain degré de standardisation de la relation siège-filiale, à travers des fonctions 
communes et des objectifs partagés. La standardisation a ici une dimension technique : elle passe par la création de procédures formelles, de mécanismes de coordination organisationnelle et décisionnelle (Van de Ven et al., 2012). Elle repose également sur de la communication informelle et le partage de normes et valeurs, faisant référence à la possibilité d'une intégration de nature normative. Cela souligne, de manière pourtant implicite, que la relation siège-filiale n'est pas unidirectionnelle. Les problématiques de RSE semblent en effet propices à des échanges allant dans les deux sens, de la filiale vers le siège et vice-versa. Plus encore, les échanges filiale-filiale ont certainement une importance particulière quand il s'agit de lancer des coopérations ou de partager des expériences.

La troisième question porte sur la variété de ces relations. Observe-t-on de nouvelles modalités d'interactions entre le groupe et ses filiales à partir de la RSE ?

\section{II - ÉTUDE DE CAS ET RÉSULTATS}

La RSE est porteuse d'enjeux transversaux qui peuvent affecter une entreprise indépen-

\section{MÉTHODOLOGIE}

L'analyse repose sur une étude de cas encastrée et longitudinale (Yin, 2004), couvrant une période de douze ans (2000-2012), permettant d'explorer des phénomènes dans leur contexte et en dynamique (Dumez, 2013). Le champ d'investigation est le groupe Bouygues. La diversification des activités permet d'analyser la prise en compte de la multiplicité des attentes et des enjeux. L'unité d'analyse, les interactions siège-filiales, permet de mettre l'accent sur les dynamiques relationnelles, à la fois entre le siège et ses cinq filiales, mais aussi, dans une moindre mesure, entre les filiales elles-mêmes.

Les matériaux collectés comprennent les rapports annuels de 2000 à 2012, des documents internes et des entretiens semi-directifs de chaque membre du comité de pilotage RSE et développement durable (DD) réunissant les filiales sous la responsabilité fonctionnelle du directeur DD au niveau du siège, et deux membres du comité de direction des filiales.

Les données récoltées ont fait l'objet d'un traitement par sériation (Dumez et Rigaud, 2008) dont le but était de produire des narrations structurées (Pestre, 2011). Des chronologies ont été réalisées en distinguant pour chaque filiale les actions à caractère plutôt environnemental ou social. Des organigrammes ont été réalisés pour situer la place du responsable RSE/DD au sein de l'organisation de la filiale. Ceci a été fait pour l'ensemble des filiales, de manière synchronique, pour l'année 2010 mais également de manière diachronique pour les filiales où les changements étaient importants sur la période 2000-2012.

Les entretiens ont fait l'objet d'un codage manuel, systématique et plurinominal (Dumez, 2013) à partir de catégories issues des guides d'entretiens et de catégories émergentes. Les données du codage permettent d'identifier les points d'inflexion ou de rupture dans les séquences chronologiques (Abbott, 2001) et d'apporter nuances et précisions lors de la construction des narrations et du sens de l'action. 
damment de la spécificité technique de ses opérations de production. Dans un groupe diversifié, un double défi apparaît alors. Celui d'adapter le langage et les pratiques aux enjeux de RSE. Celui d'articuler les enjeux, au niveau du groupe et des filiales. Quelles sont les dynamiques permettant de comprendre comment se construisent les enjeux de RSE dans un groupe où les pressions sont diversifiées par activité ? Dans quelle mesure cela fait-il évoluer l'interdépendance des entités par rapport à ces enjeux?

\section{Le groupe industriel Bouygues comme archétype du groupe industriel diversifié}

Le champ d'investigation retenu dans ce cadre est le groupe Bouygues. En tant que société cotée, la loi française lui impose de publier un rapport d'activité annuel et de le faire certifier. La notoriété du groupe et sa visibilité médiatique le rendent sensible au regard et aux critiques de diverses parties prenantes ayant le pouvoir de déclencher des conflits et de porter atteinte à la réputation de l'entreprise. Le groupe est diversifié, ce qui permet de centrer l'étude sur la prise en compte de la multiplicité des attentes et des enjeux par le siège. Trois filiales opèrent dans le secteur de la construction (immobilier, bâtiments et travaux publics, routes) tandis que deux autres relèvent de l'industrie des médias et technologies de la communication. Ceci permet de mettre en évidence les problématiques de prise en compte d'une multiplicité d'attentes et d'enjeux par le siège liées à la variété même des activités plus que par rapport à la variable géographique, comme c'est le cas dans de nombreuses études sur les multinationales (Borini et al., 2012 ; Pestre, 2010, 2011 ; Zyglidopoulos, 2002). Les filiales appartenant au métier de la construction sont par nature plus sensibles aux enjeux environnementaux, ayant désormais de forts impacts concurrentiels, tandis que les filiales médias et télécommunications sont a priori plus sensibles aux priorités sociales et humaines. En outre, la présence de plusieurs filiales appartenant à un même secteur industriel permet d'analyser les phénomènes de concurrence ou de coopération entre les filiales au regard de cette appartenance. D'un point de vue financier, Bouygues S.A., la holding, détenait en 2010 respectivement $100 \%$ du capital de Bouygues Construction et Bouygues Immobilier, 96,6 \% de Colas, 89,5 \% de Bouygues Télécoms et $43,1 \%$ de TF $1^{2}$.

Le management de la RSE et du développement durable chez Bouygues se fait au moyen d'une «double couche d'animation transverse », pour reprendre l'expression d'un membre du comité de direction de Bouygues Télécoms. Au niveau supérieur du groupe, c'est Olivier Bouygues qui est responsable de l'animation au niveau du comité de direction générale. Au niveau, inférieur, l'action est coordonnée par un directeur RSE et DD de Bouygues S.A. : il pilote les responsables RSE et DD au niveau de chaque filiale (niveau N-2 ou 3), coordonne les actions et veille à ce que les priorités établies par le comité de direction (Codir) de Bouygues S.A. soient prises en compte. Tous les trimestres, un comité mélange

2. Les chiffres proviennent du site internet du groupe et du rapport annuel 2010. 
les deux niveaux (membres du comité de direction et responsables opérationnels). La période 2000-2012 se justifie de manière pragmatique par le fait que les rapports des dix dernières années sont disponibles en ligne mais aussi par le fait que 2005 marque un tournant institutionnel important pour le groupe Bouygues. C'est à cette date qu'est créée la fonction directeur RSE/ DD au niveau du siège de Bouygues S.A., accompagnée de la mise en place du comité de pilotage interfiliale. Nous allons donc chercher à comprendre ce qui a conduit le groupe à introduire ce changement et en quoi il contribue à transformer l'organisation en interne et en externe.

\section{Résultats}

Les résultats sont présentés de manière à apporter des éléments de réponse aux trois questions posées en introduction.
Dans un groupe diversifié, où les pressions dépendent des activités :

1) Comment évaluer l'interdépendance des entités sous l'effet des enjeux de RSE ?

2) Une politique RSE est-elle un facteur de centralisation ou de décentralisation?

3) Observe-t-on de nouvelles modalités d'interactions entre le groupe et ses filiales ?

\section{L'interdépendance entre les filiales : vision statique en 2010}

À partir des entretiens, nous avons classé les enjeux par filiale ${ }^{3}$ en 2010 pour identifier les priorités partagées de celles spécifiques. À partir de cette vision statique des interdépendances, nous nuançons l'analyse en donnant une interprétation dynamique du phénomène, en distinguant les enjeux d'emblée transversaux de ceux l'étant devenus. La transversalité des enjeux communs implique pour les entités de nouvelles

Tableau 1 - Liste des enjeux majeurs par activité (le point de vue des acteurs)

\begin{tabular}{|c|c|c|c|c|c|}
\hline SA & BI & BC & Colas & BT & TF1 \\
\hline Carbone & $\begin{array}{c}\text { Construction } \\
\text { durable }\end{array}$ & $\begin{array}{c}\text { Construction } \\
\text { durable }\end{array}$ & Carbone & Carbone & $\begin{array}{c}\text { Progrès et } \\
\text { éthique }\end{array}$ \\
\hline Énergie & Énergie & Carbone & $\begin{array}{c}\text { Attractivité } \\
\text { RH }\end{array}$ & Énergie & $\begin{array}{c}\text { Diversité et } \\
\text { discrimination }\end{array}$ \\
\hline Biodiversité & Diversité & $\begin{array}{c}\text { Éthique } \\
\text { et RH }\end{array}$ & $\begin{array}{c}\text { Acceptabilité } \\
\text { sociale } \\
\text { des sites }\end{array}$ & $\begin{array}{c}\text { Diversité et } \\
\text { discrimination }\end{array}$ & Handicap \\
\hline $\begin{array}{c}\text { Diversité et } \\
\text { discrimination }\end{array}$ & $\begin{array}{c}\text { Bien-être } \\
\text { au travail }\end{array}$ & Éthique & Fournisseurs & $\begin{array}{c}\text { Recyclage, } \\
\text { Énergie, CO }\end{array}$ \\
\hline \multicolumn{7}{|c|}{} & \multicolumn{3}{|c|}{ Santé } & Fournisseurs \\
\hline
\end{tabular}

3. L'administration générale du groupe, Bouygues S.A., est désignée par l'acronyme «SA ». Les filiales ayant un nom composé sont désignées par leurs initiales. BI pour Bouygues Immobilier, BC pour Bouygues Construction, BT pour Bouygues Télécoms. 
formes de dépendance réciproque, tant dans les discours que dans les pratiques.

Les enjeux communs sont ainsi l'empreinte carbone et de l'énergie, ainsi que des problématiques de ressources humaines (RH) autour de la diversité et de la discrimination. Le bilan carbone n'a pas le même impact pour toutes les filiales. Il est très fort chez Colas, qui a développé très tôt un logiciel spécifique (« calculette carbone »), permettant de calculer le bilan carbone de projets en faisant évoluer les variables. Il est faible chez BI en interne, mais fort dans ses activités externes : en tant que promoteur, BI peut agir en aval en promouvant des bâtiments haute qualité environnementale (HQE). Historiquement, l'enjeu du bilan carbone na pas été perçu comme pertinents pour les filiales de service, par comparaison avec les filiales du BTP. Les visions ont pourtant évolué, comme le souligne la personne coordinatrice de la politique de RSE chez TF1, qui était auparavant chargée de la gestion des risques. Ainsi, elle reconnaît avoir appris à « regarder différemment l'activité 》 de TF1 et à « voir les conséquences environnementales des activités de production, les besoins en équipements technologiques, la consommation d'énergie, les frais de transports entre les différents sites des filiales ». Le sens donné aux pratiques et aux actions évolue. Certaines pratiques se constituent à mesure que les discours donnent à voir les dimensions et impacts du bilan carbone : performance énergétique des équipements technologiques, déplacements, recyclage. La variable environnementale et énergétique entre en ligne de compte dans la lecture que les acteurs font de leur activité, engendrant de nouvelles pratiques et routines. Le recyclage des équipements technologiques est instauré par le biais de partenariats avec des associations ; des solutions communes sont élaborées pour réduire les déplacements entre les différents sites de l'entreprise. En outre, dans les filiales de la construction, ils deviennent des enjeux concurrentiels, prenant ainsi une dimension stratégique. Devenus communs, ces enjeux gardent ainsi un statut différencié selon le domaine d'activité.

Les ressources humaines comprennent des enjeux de RSE, sous différentes formes, concernant l'ensemble des filiales. Chez Colas, la RSE est une opportunité pour améliorer l'attractivité de l'activité pour les jeunes diplômés. La question de la diversité parmi les cadres devient un enjeu majeur partagé aussi avec BT, BI et BC. $\mathrm{La}$ représentation et le traitement égal des minorités ethniques, des femmes et des collaborateurs seniors aux postes à responsabilité concernent l'ensemble des filiales. Le sujet est traité différemment tout en faisant l'objet d'une coordination au niveau du siège. Cette coordination par le siège est la manifestation de la dépendance mutuelle qui s'est établie en l'espace de dix ans. Les filiales sont désormais tenues d'agir de manière concertée, elles doivent répondre de leurs actions et progrès au siège sur un ensemble de questions nouvelles, tandis que le groupe, en tant que société cotée, est tenu pour légalement et socialement responsable des discours et agissements de ses filiales, via la pratique du reporting. L'interdépendance entre les entités du groupe s'est ainsi élargie aux questions environnementales et sociales sous la pression d'enjeux communs qui étaient bien souvent des «non-questions" pour les filiales. Quelles sont alors les caractéristiques des dynamiques collectives impulsées par cette nouvelle situation? 


\section{L'intégration de la RSE dans un groupe diversifié : facteur de centralisation ou de décentralisation?}

Peut-on identifier une dynamique commune pour la relation siège-filiale, où la RSE serait un facteur de centralisation ou de décentralisation? Nous avons mis en évidence le débat entre l'hypothèse de la théorie de la contingence (Donaldson, 2001 ; Doz et Prahalad, 1984 ; Nohria et Ghoshal, 1994) et l'hypothèse inverse défendue par les tenants de la stratégie de centralisation (Cruz, et al., 2010 ; Cruz et Pedrozo, 2009 ; Daudigeos, 2009). L'analyse repose sur une chronologie fine de la période 2000-2011 réalisée à partir des rapports d'activité et des résultats du codage des entretiens. L'année 2005 constitue un point de référence, car c'est à ce moment qu'a été créée la fonction de directeur développement durable au niveau de Bouygues S.A. C'est un événement important car il officialise la prise en compte au plus haut niveau du groupe des enjeux de DD et RSE, mais l'on peut se demander si cette période critique constitue une épiphanie ${ }^{4}$ (Denzin, 1989) ou un bouleversement apparent. Avant 2005, Bouygues avait une division environnement, le groupe Saur, et des responsables qualité, sécurité, environnement dans les filiales. Dans les entretiens, 2005 est associée à un changement important, les acteurs la considérant comme un tournant dans la gestion du DD et de la RSE au niveau du groupe suite à une initiative du PDG du groupe. Les acteurs perçoivent la période d'avant 2005 comme une période de transition : en 2000, l'heure était à la gestion des risques et les changements se font de manière graduelle, constituant des points d'inflexion.

Dans les rapports d'activité 2000 et 2001, les filiales communiquant sur leur action environnementale sont BT (installation des antennes radio), BC (démantèlement et désamiantage, conception de bâtiments verts, gestion des déchets) et Colas (réhabilitation des carrières, gestion des déchets, gestion des sites de production et chantiers, pollutions). TF1 et BI sont absentes. Colas est la plus en pointe, en ayant mis en œuvre des politiques de gestion technique et instrumentée des questions environnementales (amélioration des performances environnementales des produits, conformité réglementaire). Le DD entre dans l'entreprise en étant articulé avec la gestion des risques et l'harmonisation du système de management qualité, sécurité, environnement (QSE) au niveau du groupe. En 2001, est adoptée « une politique qualité, sécurité, environnement, en ligne avec les principes d'excellence qui structurent les normes internationales de management $»$. Dans le rapport 2002, les informations concernant l'environnement sont désormais synthétisées thématiquement pour le groupe, chaque filiale étant mentionnée en fonction de ses actions. La gestion des ressources humaines, faisant l'objet d'une page factuelle dans le rapport 2000, devient un sujet politique. Un discours élaboré au niveau du groupe sur «les hommes, une valeur forte » permet l'entrée de thèmes comme le dialogue social, la formation, la parité, les questions de motivation et fidélisation.

4. Une épiphanie entraîne une évolution radicale des modes de pensée et d'action des acteurs suite à un changement dans le réel. 
On observe ainsi un décalage entre les problématiques environnementales et celles de gestion des hommes. Les premières font l'objet d'actions précoces visant une homogénéisation des pratiques (mise en œuvre de normes techniques ayant un impact sur les pratiques de management et les processus de production) tandis que les secondes commencent à faire l'objet d'un discours politique fondé sur la mise en avant de valeurs, signe d'un léger découplage (Bromley et Powell, 2012). L'attention portée à la simple structure des rapports et leur évolution chronologique montre que la période constitue pour le groupe une phase critique que l'on pourrait qualifier de point d'inflexion en ce que les changements se font de manière graduelle, sans être toujours explicités et ne restent pas dans la mémoire des acteurs interrogés comme étant centraux.

En 2005, cette évolution progressive se cristallise par la création de la fonction de directeur développement durable au niveau $\mathrm{du}$ siège. Ceci marque un changement organisationnel fort dans la mesure où les filiales sont directement touchées par la création du comité transversal. C'est un premier élément formel de standardisation de la relation faisant suite à la préconisation d'harmonisation des systèmes de management QSE mais formalisant la séparation des deux activités. Une dynamique de coordination s'instaure : la fonction RSE ou DD est créée dans chaque filiale, mais elle ne s'accompagne pas d'une standardisation du statut, des rôles et missions de la fonction au sein de chaque filiale.

L'épiphanie de 2005 (Denzin, 1989) est alors suivie par une phase de changements liée à la prise en compte d'attentes sociales nouvelles (2005-2011). La diffusion de connaissances sur les impacts environnementaux des activités provoque des changements d'attitudes et de croyances allant au-delà de la mise en œuvre de procédures techniques QSE. En outre, en tant qu'entreprise multinationale leader dans plusieurs activités, Bouygues est soumise à des demandes politiques croissantes concernant les enjeux de gouvernance. Le PDG du groupe, Martin Bouygues, est ainsi amené à signer le Pacte mondial en 2006 suite à une demande formulée par le Président de la République Jacques Chirac. Des projets sont impulsés, les pratiques de management se standardisent dans une certaine mesure, comme dans le cas du reporting - un même format de tableau pour les filiales est publié à partir du rapport 2007, impliquant une démarche de progrès continu et d'autoévaluation, une homogénéisation des catégories d'indicateurs classant les actions dans trois pôles (économique, environnemental, social) reprenant ainsi le thème (créé par John Elkington) de la «triple bottom line » et réduisant ainsi le décalage de traitement discursif et pratique entre l'environnemental et le social.

Pendant cette période, toutefois, les bouleversements ne sont ni radicaux ni uniformes. Après une phase d'homogénéisation, la dynamique centralisatrice n'est pas allée jusqu'à l'intégration dans une stratégie unique établie au niveau du groupe pour être déclinée à l'identique. Ainsi, le siège n'intervient pas dans le choix organisationnel des filiales quant au département de rattachement de la fonction RSE-DD. On observe une certaine instabilité organisationnelle, des tâtonnements et des relations conflictuelles entre les filiales, analysées dans la partie suivante. Les relations entre le siège et ses filiales ne peuvent faire l'ob- 
jet d'une analyse linéaire et uniforme. Le discours et les pratiques montrent un traitement centralisé des enjeux de DD et de RSE au niveau du groupe, mais il faut préciser que l'homogénéisation des pratiques est inégale entre les filiales et entre les sujets. 2005 apparaît ainsi un tournant entre une phase de centralisation, puis une phase marquée par des tâtonnements et de l'instabilité organisationnelle. Dans un troisième temps, nous nous intéressons à cette dynamique d'instabilité et à ses manifestations. Nous mettons en évidence de nouvelles modalités d'interaction entre le groupe et ses filiales et leurs enjeux.

\section{De nouvelles modalités relationnelles entre siège et filiales mélangeant standardisation technique et intégration normative}

La dynamique de centralisation s'accompagne-t-elle d'une standardisation formelle des relations? L'analyse penche en faveur d'un mélange d'intégration formelle (par des techniques et outils de gestion) et informelle (par les normes et les valeurs). Une manière de rendre visible le degré de standardisation de la relation siège-filiale a consisté à comparer les organigrammes des filiales en synchronique mais aussi de manière diachronique.

On observe que les responsables opérationnels du DD et de la RSE ont des niveaux différents en fonction des filiales et que leur rattachement peut évoluer dans le temps. Dans certaines filiales, comme Colas, l'intitulé du poste est ainsi resté «Directeur environnement » avant et après 2005, les dimensions non environnementales de la RSE entrant dans le champ de compétences du directeur sans modifier la prépondérance historique des questions environnementales. Ce directeur a un niveau hiérarchique relativement élevé par rapport aux autres filiales puisqu'il dépend directement de l'administrateur du siège du groupe, et non d'un directeur général (DG) spécifique. Le poste le plus sous-dimensionné est chez Bouygues Télécoms (BT) où la responsable est au niveau $\mathrm{N}-3$, répondant au directeur QSE$^{5}$. Toutefois, le DG en charge des questions de RSE s'implique personnellement dans la définition de la stratégie DD-RSE et dans la réflexion prospective, signifiant que ces questions ne sont pas minimisées par la filiale mais portées à deux niveaux différents, opérationnel et stratégique.

Cette différenciation par filiale est reflétée dans les intitulés de poste. Dans les filiales où le $\mathrm{DD}$ se traduit en enjeux concurrentiels (Colas, BC et BI), alors un directeur est chargé de la gestion de ces derniers. En revanche, chez BT et TF1, la fonction revient respectivement à une « responsable » et une « coordinatrice ».

Au-delà des intitulés, le périmètre d'action et les changements de tutelle fournissent d'autres éléments pour analyser la permanence de l'instabilité organisationnelle. Pour BI, le template diachronique (20082012) montre que la fonction était initialement sous tutelle du marketing, avant de passer à la filière métier. Après avoir eu une dimension QSE appuyée (même direction générale, et une directrice ingénieure QSE de formation), c'est la dimension innovation qui prend de l'importance en 2010. La direction DD avait alors une mission de coordination et de pilotage transversal

5. Cette affiliation montre que la différenciation des deux filières, QSE et RSE-DD, est loin d'être complète. 
Figure 1 - Organigrammes synchroniques des cinq filiales (2010)

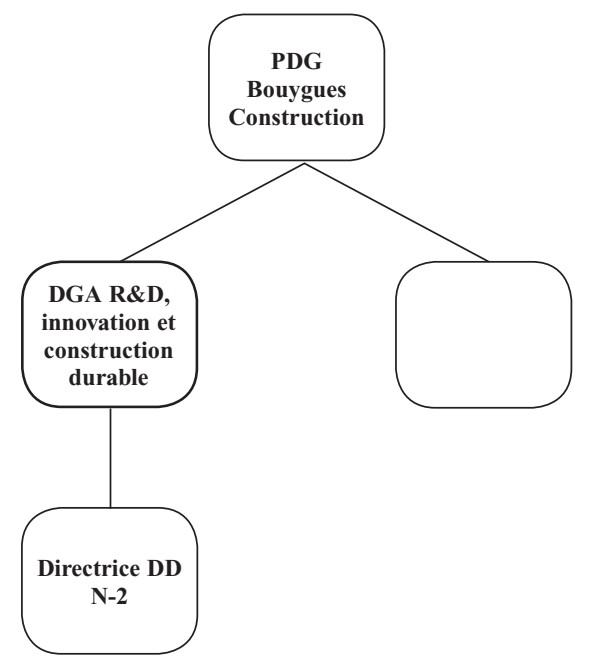

Bouygues Construction 2010

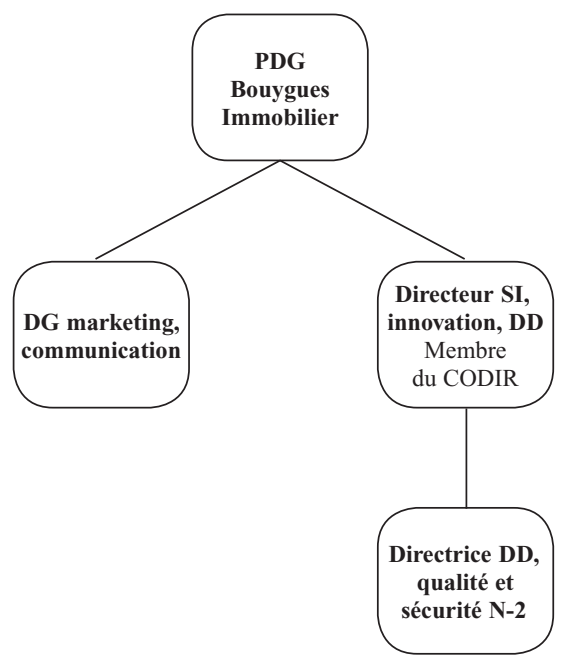

Bouygues Immobilier 2010

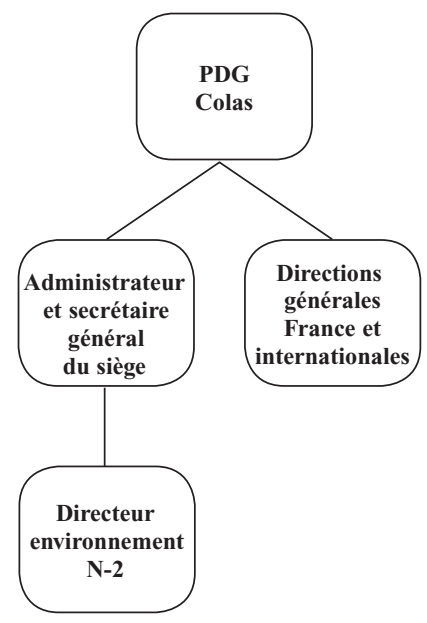

Colas 2010

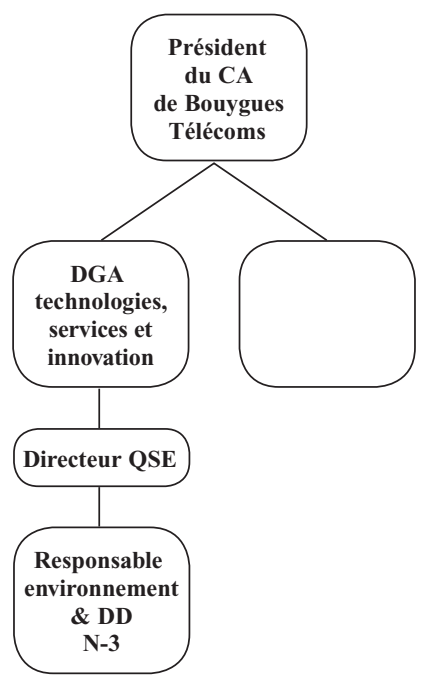

Bouygues Télécom 2010

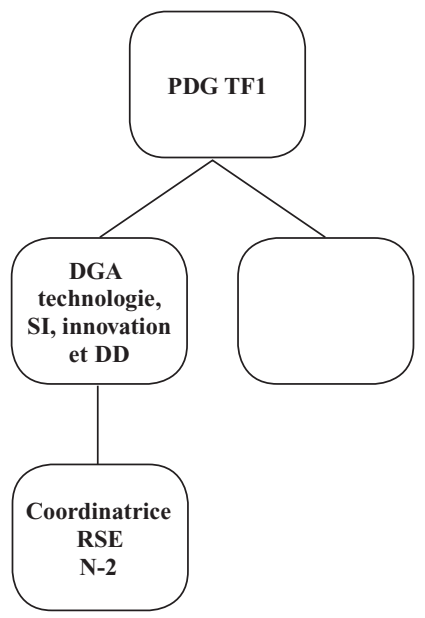

TF1 2010 
et rendait compte au comité exécutif. En 2012, pourtant, la direction DD repasse sous la tutelle d'une direction transversale communication, marketing et DD, avec une nouvelle directrice dont le parcours s'est essentiellement déroulé dans le management du développement durable avec une orientation marketing.

Deux observations concernent les trois filiales opérant dans la construction. Chaque filiale réfléchit de façon autonome au design organisationnel et à l'intégration stratégique des enjeux de RSE liés à l'évolution du marché. L'instabilité organisationnelle suggère que la centralisation ne passe pas par la mise en place de mécanismes formels de contrôle. Quand certains enjeux interprétés par les dirigeants comme relevant du DD se transforment en enjeux concurrentiels forts, alors l'organisation s'adapte et est adaptée. La «construction durable» acquiert une autonomie à la fois technique et économique en étant reliée au cœur de métier des filiales $\mathrm{BC}$ et $\mathrm{BI}$, tandis que les enjeux de RSE dans leur ensemble restent du ressort de la direction DD, au rôle transversal (reporting) et en charge de l'animation managériale aux différents niveaux de l'organisation. Ceci pourrait en partie s'expliquer par les tensions entre les filiales partiellement en situation de concurrence interne et de ce fait pas toujours promptes à mutualiser leurs savoirs ou leurs compétences :

«Nous avons une démarche structurée (Actitudes 42 actions) qui existe moins dans les autres métiers. Urbanaria devenu Ecocity met en commun les savoirs des métiers pour imaginer les éco-quartiers de demain. Il y a encore des tensions entre Bouygues Immobilier et Bouygues Construction. Il n'y a pas d'envie de partage des savoirs. BI a fait Green Office sans BC. ${ }^{6}$ »

Figure 2 - Organigramme diachroniques de Bouygues Immobilier (2008-2012)

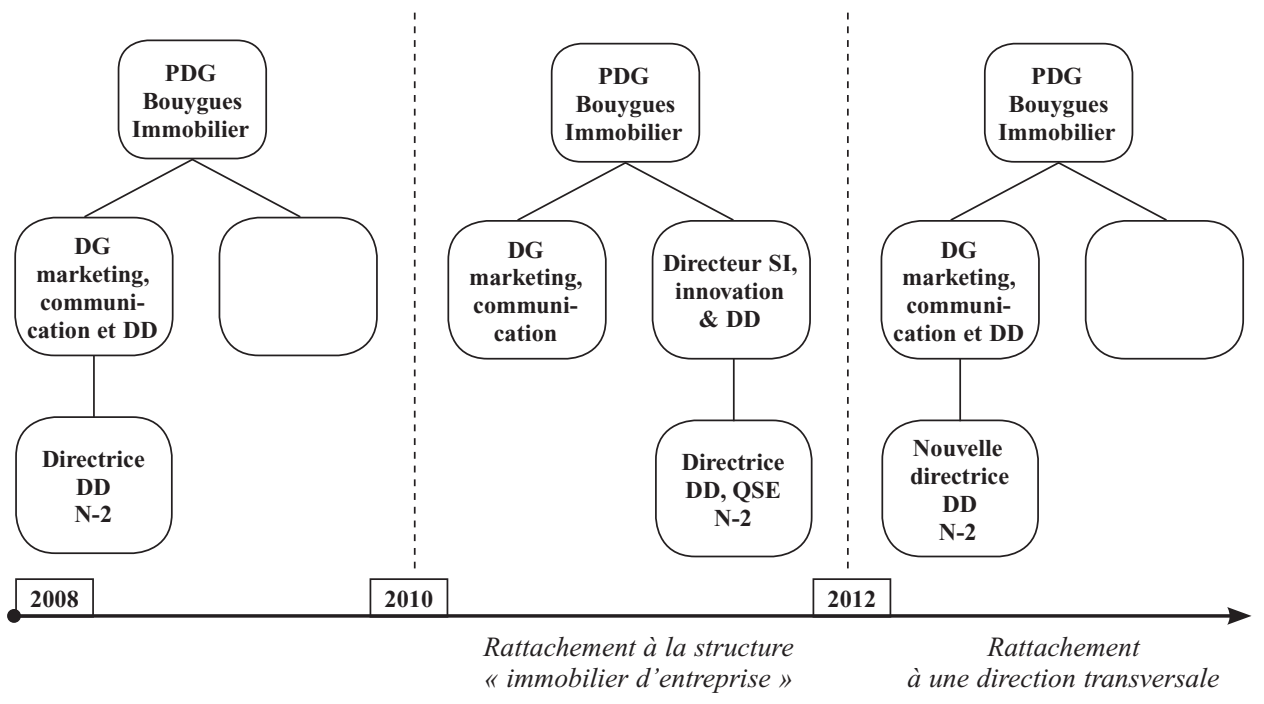

6. Entretien Bouygues Construction. 
Étonnamment, on observe plus de stabilité organisationnelle dans les activités de télécommunications et de services, malgré une sensibilité initiale inégale au départ. BT est concerné depuis sa création par les questions environnementales et de santé (antennes relais), tandis que les enjeux forts pour TF1 sont historiquement les sujets $\mathrm{RH}$ et les relations avec la société civile. Cependant, dans les deux cas, la fonction RSE et DD a été placée sous la tutelle de la direction générale technologies, services et innovation. Chez BT, les personnes interrogées reconnaissent le caractère « opportuniste » de ce rattachement : il n'y a donc pas eu, au préalable, de réflexion concertée entre le siège et ses filiales ni d'imposition.

In fine, les modes de contrôle du siège restent assez souple, passant avant tout par la promotion de normes et d'outils de soft law. Le code d'éthique se veut un guide pour l'action de chaque collaborateur, en toutes circonstances; il est accompagné de chartes communes (charte des ressources humaines, charte sociale européenne, charte RSE des relations avec les fournisseurs) et de divers engagements (pacte mondial) reposant sur des principes et des valeurs. Le contrôle formel, quant à lui, passe par la mise en œuvre d'un reporting harmonisé et de critères de RSE dans l'évaluation des managers.

\section{III - CONTRIBUTIONS, LIMITES ET PERSPECTIVES D'APPROFONDISSEMENTS}

À ce point de la recherche plusieurs résultats ont été mis en avant. Les enjeux de RSE créent des interdépendances entre les activités et cela s'est concrétisé dans le groupe par la création d'une structure de management commune pour coordonner les actions de RSE. Ces dernières restent en revanche spécifiques aux métiers, en fonction des impacts de chaque enjeu sur l'activité. Des mécanismes de coordination et de gestion centralisée de la relation entre le siège et les filiales sur les questions de RSE se sont développés, autour des pratiques de reporting, de l'évaluation des managers et de la mise en œuvre d'une « double couche d'animation transverse » alliant la coordination fonctionnelle à des niveaux hétérogènes et le contrôle hiérarchique par un membre du comité de direction de chaque filiale.

Les analyses montrent de nombreux tâtonnements, au niveau des filiales et du groupe. L'instabilité organisationnelle demeure, malgré la mise en place de procédures et d'objectifs communs. Les relations entre le siège et ses filiales ne font pas l'objet d'une centralisation standardisée formellement, au-delà de la mise en place du reporting et des critères d'évaluation des managers. Les politiques communes s'appuient sur des instruments normatifs diffusés dans les différentes activités et créant des référentiels communs. Les relations de tension entre certaines filiales opérant sur des marchés proches, voire sécants, est un frein aux pratiques de coopération dans le groupe.

Les contributions théoriques portent sur la relation siège-filiale dans le cas de l'implantation de politiques transverses de RSE. La diversité des activités industrielles semble favoriser des modalités de contrôle souple et normatif par un siège plus qu'une centralisation complète et formelle, accompagnée de modalités relationnelles uniformes et standardisées. L'impact concurrentiel 
de certains enjeux environnementaux ou sociétaux peut constituer un facteur majeur d'instabilité dans la relation siège-filiale et filiale-filiale et freiner une coopération poussée entre les entités d'un groupe et la mise en œuvre de programmes intégrés.

Ce cas propose ainsi une situation intéressante dont il est nécessaire d'approfondir plusieurs aspects pour comprendre ce qui est sous-jacent à l'instabilité organisationnelle observée. Pour étudier plus précisé- ment la dynamique centralisation/décentralisation, il serait judicieux de poursuivre l'étude dans le temps et de proposer une étude plus systématique des phénomènes de couplage et découplage à la fois entre discours et pratiques, et entre moyens et fins (Bromley et Powell, 2012). À un autre niveau d'analyse, il serait utile, également, de descendre au niveau de la gestion des enjeux de RSE dans les filiales de chaque branche du groupe.

\section{BIBLIOGRAPHIE}

Abbott A. (2001). Time matters: On theory and method, University of Chicago Press, Chicago, IL.

Abdellatif M. (2011). «Le contrôle des filiales à l'étranger», Revue française de gestion, vol. 37, n²12, p. 157-170.

Ackerman R.W. et Bauer R.A. (1976). Corporate social responsiveness: the modern dilemna, Reston Publications, Reston, VA.

Acquier A., Daudigeos T. et Valiorgue B. (2011). "Corporate social responsibility as an organizational and managerial challenge: the forgotten legacy of the Corporate Social Responsiveness movement”, M@n@gement, vol. 14,n4, p. 221-250.

Aggeri F. (2011). "Le développement durable comme champ d'innovation », Revue française de gestion, vol. 37, $\mathrm{n}^{\circ}$ 215, p. 87-106.

Amba-Rao S.C. (1993). "Multinational corporate social responsibility, ethics, interactions and Third World governments: An agenda for the 1990s", Journal of Business Ethics, vol. $12, \mathrm{n}^{\circ} 7$, p. 553-572.

Bastianutti J. et Dumez H. (2012). « Pourquoi les entreprises sont-elles désormais reconnues comme socialement responsables ? ", Gérer et comprendre, ${ }^{\circ} 109$, septembre, p. 44-54.

Beddi H. (2011). "Quel est le rôle du siège dans les firmes multinationales ? ", Revue française de gestion, vol. 37, $\mathrm{n}^{\circ} 212$, p. 77-92.

Birkinshaw J., Holm U., Thilenius P. et Arvidsson N. (2000). "Consequences of perception gaps in the headquarters-subsidiary relationship”, International Business Review, vol. 9, $\mathrm{n}^{\circ} 3$, p. 321-344.

Borini F.M., Oliveira Jr. M.M., Silveira F.F. et Concer R.O. (2012). "The reverse transfer of innovation of foreign subsidiaries of Brazilian multinationals", European Management Journal, vol. 30, n³, p. 219-231. 
Bromley P. et Powell W.W. (2012). "From smoke and mirrors to walking the talk: Decoupling in the contemporary world", The Academy of Management Annals, vol. 6, n 1, p. 483-530.

Crozier M. et Friedberg E. (1977). L'acteur et le système, Le Seuil, Paris.

Cruz L. B. et Pedrozo E. A. (2009). "Corporate social responsibility and green management: Relation between headquarters and subsidiary in multinational corporations", Management Decision, vol. 47, $\mathrm{n}^{\circ}$ 7, p. 1174-1199.

Cruz L.B., Pedrozo E.A., Barros Estivalete V. et Nayar Hoff D. (2010). "The influence of transverse CSR structure on headquarters/subsidiary integration", Brazilian Administration Review, vol. 7, n 3, p. 310-324.

Daudigeos T. (2009). «Rendre l'entreprise néolibérale responsable. Rôle des logiques institutionnelles et des experts fonctionnels. Étude de la gestion du risque accident du travail dans le secteur de la construction », Thèse soutenue à l'Université Jean Moulin Lyon III, 25 novembre.

Denzin N.K. (1989). The research act: a theoretical introduction to sociological methods, Prentice Hall Englewood Cliffs, N.J.

Donaldson L. (2001). The contingency theory of organizations, Sage Publications, Thousand Oaks, CA.

Doz Y. et Prahalad C.K. (1984). "Patterns of strategic control within multinational corporations”, Journal of International Business Studies, vol. 15, n 2, p. 55-72.

Dumez H. (2013). Méthodologie de la recherche qualitative: les 10 questions clés de la démarche compréhensive, Vuibert, Paris.

Dumez H. et Rigaud E. (2008). «Comment passer du matériau de recherche à l'analyse théorique : à propos de la notion de "template" ", Le Libellio d'Aegis, vol. 4, n 2, p. $40-46$.

Hamilton III R.D., Taylor V.A. et Kashlak R.J. (1996). "Designing a control system for a multinational subsidiary", Long Range Planning, vol. 29, n 6, p. 857-868.

Martinez J.I. et Jarillo J.C. (1991). "Coordination demands of international strategies", Journal of International Business Studies, vol. 22, n 3, p. 429-444.

Mayrhofer U. (2011). « La gestion des relations siège-filiales », Revue française de gestion, vol. $37, \mathrm{n}^{\circ} 212$, p. $65-75$.

Muller A. (2006). “Global versus local CSR strategies", European Management Journal, vol. 24, n²-3, p. 189-198.

Nohria N. et Ghoshal S. (1994). "Differentiated fit and shared values: Alternatives for managing headquarters-subsidiary relations", Strategic Management Journal, vol. 15, p. 491-502.

Pestre F. (2010). « Comment l'entreprise multinationale peut-elle lutter contre la pauvreté ? », Revue française de gestion, vol. 36, n²08-209, p. 137-154. 
Pestre F. (2011). «Construire une stratégie de responsabilité sociale de la firme multinationale », Revue française de gestion, vol. 37, $\mathrm{n}^{\circ} 212, \mathrm{p} .109-125$.

Pinkston T.S. et Carroll A.B. (1992). "Corporate citizenship: a comparative analysis of foreign affiliates located in the U.S. and their domestic counterparts", Academy of Management Best Papers Proceedings, p. 353-357.

Rao H. et Sivakumar K. (1999). "Institutional sources of boundary-spanning structures: The establishment of investor relations departments in the Fortune 500 Industrials", Organization Science, vol. $\mathrm{n}^{\circ} 10,1$, p. 27-42.

Richter A., West M., Van Dick R. et Dawson J. (2006). “Boundary spanners' identification, intergroup contact, and effective intergroup relations", The Academy of Management Journal Archive, vol. 49, n 6, p. 1252-1269.

Roth K. et Nigh D. (1992). "The effectiveness of headquarters-subsidiary relationships: The role of coordination, control, and conflict”, Journal of Business Research, vol. 25, $\mathrm{n}^{\circ} 4$, p. 277-301.

Schotter A. et Beamish P.W. (2011). "Performance effects of MNC headquarters-subsidiary conflict and the role of boundary spanners: The case of headquarter initiative rejection", Journal of International Management, vol. 17, $\mathrm{n}^{\circ}$ 3, p. 243-259.

Van de Ven A.H., Leung R., Bechara J.P. et Sun K. (2012). "Changing organizational designs and performance frontiers", Organization Science, vol. 23, n 4, p. 1055-1076.

Veugelers R. et Cassiman B. (2004). "Foreign subsidiaries as a channel of international technology diffusion: Some direct firm level evidence from Belgium”, European Economic Review, vol. 48, n², p. 455-476.

Williams P. (2002). “The competent boundary spanner", Public Administration, vol. 80, n 1, p. 103-124.

Yin R.K. (2004). Case study research: design and methods, $3^{\mathrm{e}}$ édition, Sage Publications, Thousand Oaks, CA.

Yu T. et Lester R.H. (2008). "Moving beyond firm boundaries: A social network perspective on reputation spillover”, Corporate Reputation Review, n 11, p. 94-108.

Zyglidopoulos S.C. (2002). "The social and environmental responsibilities of multinationals: Evidence from the Brent Spar case", Journal of Business Ethics, vol. 36, n 1-2, p. 141-151. 